\title{
$\begin{array}{ll}\text { Research Square } & \text { Preprints are preliminary reports that have not undergone peer review. } \\ \text { They should not be considered conclusive, used to inform clinical practice, }\end{array}$
}

\section{A Single Knotless-anchor for Bursal-side Ellman III Partial Thinkness Rotar Cuff Tears: A Biomechanical Cadaveric Study}

\section{Gang Liu}

Affiliated Traditional Chinese Medicine Hospital of Southwest Medical University https://orcid.org/0000-0002-1764-5417

\section{Baolu Zhang}

College of Nursing Affiliated to Southwest Medical University

\section{Qian Zhao}

Luzhou Hospital of Traditional Chinese Medicine

\section{Xiaoguang Guo}

Affiliated Traditional Chinese Medicine Hospital of Southwest Medical University

\section{Yang Liu}

Affiliated Traditional Chinese Medicine Hospital of Southwest Medical University

\section{Bo Qin}

Affiliated Traditional Chinese Medicine Hospital of Southwest Medical University

\section{Ping Xu}

Affiliated Traditional Chinese Medicine Hospital of Southwest Medical University

\section{Tao Liang}

Affiliated Traditional Chinese Medicine Hospital of Southwest Medical University

Shijie Fu ( $\sim$ fushijieggj@126.com )

Affiliated Traditional Chinese Medicine Hospital of Southwest Medical University https://orcid.org/0000-0002-2168-6094

\section{Research article}

Keywords: Bursal-side Ellman III, Single Knotless-anchor, Double-row repair, Biomechanical study

Posted Date: January 11th, 2021

DOl: https://doi.org/10.21203/rs.3.rs-141471/v1

License: (c) (1) This work is licensed under a Creative Commons Attribution 4.0 International License. Read Full License 


\section{Abstract}

Background: Bursal-side partial thickness rotator cuff tears (PTRCTs) are frequent lesions with the treatment of multiple surgical techniques. However, to the best of our knowledge, no single knotlessanchor with two Ethicon 2\# repair technique has been reported.

Methods: Bursal-side PTRCTs (Ellman type III, 75\% thickness of tears) were created in the supraspinatus tendon on 16 fresh-frozen cadaveric shoulders. The specimens were randomized into two groups of 8 each: (1) Group A (Transtendon repairs), a single knotless-anchor repair with two Ethicon 2\#, (2) Group B, the Conversion repair (Double-row, DR). Each specimen underwent cyclic loading test from 5 to $100 \mathrm{~N}$ by 50 cycles, then followed by an ultimate failure test. The displacement of greater tuberosity $(\mathrm{mm})$ and ultimate $(\mathrm{N})$ were recorded.

Results: Compared with the load-to-failure test, there was no significant difference between Group $A$ and B (Group A, 359.25 $\pm 17.91 \mathrm{~N}$; Group B, $374.38 \pm 13.75 \mathrm{~N}, \mathrm{P} \otimes 0.05)$. There were also no significant differences in the rotator cuff displacement of $10 \mathrm{~mm}$ (Group A, 190.50 $\pm 8.52 \mathrm{~N}$; Group B, 197.25 $\pm 6.84, P \llbracket 0.05$ ) and

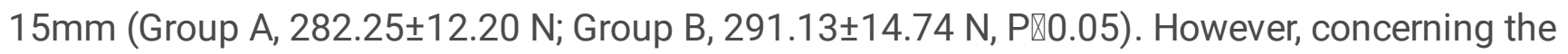
displacement of $3 \mathrm{~mm}$ and $5 \mathrm{~mm}$, we found a significant difference in these two groups $(P<0.05)$.

Conclusions: A single knotless-anchor with two Ethicon 2\# for Bursal-side Ellman III PTRCTs is a simple, cheap, and effective technique that we could choose.

\section{Background}

The rotator cuff plays an important anatomic role in shoulder movement. PTRCTs are a common lesion with a symptom such as pain or dysfunction [1, 2]. The overall prevalence of these tears ranges from 13$37 \%$, which may be related to its strong correlation with age $[3,4]$. In patients over the age of 60 , the prevalence was approximately was $26 \%$ [5]. The Ellman classification system (articular, bursal, and intratendinous) is commonly used to describe PTRCTs [6].

Despite their high prevalence, PTRCTs have been already ignored, especially in bursal-side tears. Most of the studies in treating rotator cuff tears have focused on full-thickness tears. There are two basic choices commonly used to treat this lesion: non-operative treatment and operative treatment. On the one hand, rotator cuff repair is required in a patient who diagnosis with PTRCTs (Ellman type III) [7, 8]. On the other hand, the surgical indication for PTRCTs is generally in patients who failed with non-operative treatment for 3-6 months or in younger patients suffering from a traumatic injury [9]. There were multiple surgical treatments, including arthroscopic debridement with or without acromioplasty and arthroscopic repair to this lesion. The specific strategies for repair include conversion repair and in situ repairs [10-13].

Conversion repair with double-row was common used to treat bursal-side PTRCTs, which had successful clinical and anatomic outcomes with significant improvement in range of movement, strength, and pain relief [14]. Also, it costs a lot without reimbursement. However, in our hometown, the South-west of China, 
millions of patients cannot bear medical expenses and have inferior health insurance. We are trying to find a simple and cheap way to solve this problem for those poor patients who need this surgery. In our clinical data findings, a single knotless-anchor repair, one of the transcendent repairs, was also presented with good clinical outcomes. So, the purpose of this study was to analyze whether a single knotlessanchor with two Ethicon 2\# (Johnson, USA, a high polymer polyethylene) had the same mechanical properties compared with a double-row repair.

Our study's primary hypothesis was that the biomechanical of a single knotless-anchor with two Ethicon 2\# is cheap and reliable, and we could choose this surgical technique in our hometown, South-west of China.

\section{Methods}

\section{Specimen Selection}

All donors with a mean age of 51.20 years (33 to 65) and his/her family had given written consent for body donation to this science study. 16 fresh-frozen cadaveric shoulder specimens (10 right and 6 left; 9 men and 7 women) were employed in our research. The bone density was tested by X-ray to be a normal specimen (OSTEOCORE-3, Golden, China).

For inclusion, criteria were as follows: (i) No history and signs of the previous scapula or clavicle fracture; (ii) Normal and full-grown Chinese shoulder joint; (iii) No history of the shoulder dislocation and operation; The exclusion criteria were as follows: (i) Incomplete specimens; (ii) History of drug abuse. (iii) Shoulder diseases (e.g., rheumatoid arthritis, Tuberculosis of the shoulder joint, or RCT).

\section{Bursal-side PTRCTs modeling}

All soft tissues on the scapula and humerus were resected from the bone, with only the intact supraspinatus and infraspinatus tendon retained. The humeral mid-shaft was cut approximately $20 \mathrm{~cm}$ from the top of the greater tuberosity. A length of over $5 \mathrm{~cm}$ of the supraspinatus and infraspinatus were preserved at the footprint insertion. We measured the width of the anterior and posterior and thickness of the supraspinatus tendon at the footprint using a digital caliper (Sang-Liang, japan). Then, we created $75 \%$ Bursal-side PTRCTs (Fig. 1.A) for which repair was commonly advocated using a blade $[15,16]$. To restore the injury mechanism of acromion impingement syndrome, we also used a bone contusion (liJian, Zhe Jiang, China) to create an impact on this tear (Fig. 2.B).

\section{Model repair technique}

All the distances were carefully measured by the use of calipers(Group A, Fig. 2.A-F). This repair technique used a total of one 4.75-mm Knotless-anchor (Arthrex Inc, Naples, FL, USA) and 2 Ethicon 2\# (Johnson, USA, a high polymer polyethylene). For the first suture, we used a suture hook to puncture at a distance of $3 \mathrm{~mm}$ from the upper edge of the injury. The stitch length was $2 / 3$ of the injury distance, and the PDS thread is used to pass through the hook. For the second suture, we punctured at the upper 
distance of $2 \mathrm{~mm}$ from the first one. A lateral Knotless-anchor was then placed $1 \mathrm{~cm}$ to $2 \mathrm{~cm}$ distal to the lateral edge of the footprint in line with the anchors from the first suture(Figure 2. F囚. All operations were performed by the senior Author (Shi Jie-Fu, shoulder master).

The conversion repair involved completing a PTRCTs to a full-thickness rotator cuff tear followed by repair (Fig. 2.G-H). This repair technique used a total of one 4.5-mm Knotless-anchor (Arthrex Inc, Naples, FL, USA) and one suture anchor with Three \# 2 (5 metrics) Hi-Fi sutures (ConMed Linvatec, Utica, NY, USA). After the tear's conversion, one suture anchor was used for the medial row, and one Knotlessanchor was used for the lateral row using the same techniques as described previously (Fig. 2H) [17]. Finally, we sutured both edges of the supraspinatus by using the 2 Ethicon $5 \#$ (Johnson, USA) as a specific description of Fig. 3. A.

\section{Load test}

Firstly, we secured the proximal humerus with eight screws in a special device(Figure 3. A). The supraspinatus was then secured at $90^{\circ}$ of abduction. The other side of the specimen was secured to the biomechanical testing machine (Bose Electro Force 3520-AT, USA, Fig. 3). Moreover, care was taken to ensure equal and symmetric tension on the tendon before clamping. A small device supplemented with 2 Ethicon $5 \#$ was used to secure the supraspinatus tendon. A superior preload of $100 \mathrm{~N}$ was then performed to test the specimen fixation stability, time effect, and stress relaxation. The electrodynamic testing machine was applied to the load at a constant $5 \mathrm{~N} / \mathrm{s}$ speed. 5 to $100 \mathrm{~N}$ for 50 cycles load test was repeated, and the loading interval of each test was kept $1 \mathrm{~min}$ to relieve the stress fatigue. All tests were fished in a half-day at $22^{\circ}$ temperature, and the surface of the prepared-modal was always kept moist with the use of isotonic saline.

\section{Load to failure test}

We tested the ultimate failure load $(\mathrm{N})$ with a constant speed of $1 \mathrm{~mm} / \mathrm{min}$ in the superior-inferior direction, and the mode of failure was recorded for every specimen. The supraspinatus displacement at $3 \mathrm{~mm}, 5 \mathrm{~mm}, 10 \mathrm{~mm}$, and $15 \mathrm{~mm}$ was recorded. The failure was defined as the supraspinatus rupture, Knotless-anchor or suture anchor failure, or Ethicon $5 \#$ broken.

\section{Statistical Analysis}

Statistical package for social sciences was performed by SPSS 19.0 software (Chicago, IL, USA). All data were presented by means \pm standard deviation $(\overline{\mathrm{x}} \pm s)$. The intra-group homogeneity of variance was performed with a one-way analysis of variance (one-way ANOVA) test. The independent-Sample T-test was analyzed between the two groups. A significance level of $p<0.05$ was accepted as a statistically significant difference.

\section{Results}

\section{The displacement of the supraspinatus}


The finding at $3 \mathrm{~mm}, 5 \mathrm{~mm}, 10 \mathrm{~mm}$, and $15 \mathrm{~mm}$ of the supraspinatus displacement was summarized in Table 1. Overall, a steady and significant increase could be seen in both groups $A$ and $B(P<0.05)$. Additionally, compared with groups $A$ and $B$, there was no statistically significant difference at $10 \mathrm{~mm}$ and $15 \mathrm{~mm}$ of supraspinatus displacement $(P>0.05)$. However, there was a statistically significant difference at $3 \mathrm{~mm}$ and $5 \mathrm{~mm}$ of supraspinatus displacement $(P<0.05)$.

Table 1

The displacement of supraspinatus at $3 \mathrm{~mm}, 5 \mathrm{~mm}, 10 \mathrm{~mm}$, and $15 \mathrm{~mm}$ was as follows

\begin{tabular}{|c|c|c|c|c|}
\hline Groups & $3 \mathrm{~mm}$ & $5 \mathrm{~mm}$ & $10 \mathrm{~mm}$ & $15 \mathrm{~mm}$ \\
\hline A & $84.38 \pm 6.52^{\mathrm{abcd}}$ & $130.13 \pm 11.12^{\mathrm{acd}}$ & $190.50 \pm 8.52^{d}$ & $282.25 \pm 12.20$ \\
\hline B & $100.25 \pm 6.39^{\mathrm{bcd}}$ & $144.25 \pm 10.39 \mathrm{~cd}$ & $197.25 \pm 6.84^{d}$ & $291.13 \pm 14.74$ \\
\hline
\end{tabular}

\section{Load to failure}

Although the higher mean strength $(\mathrm{N})$ of these two groups was the conversion repair (Double-row, Group $\mathrm{B}, 374.38 \pm 13.75 \mathrm{~N}$ ), there was no significant difference between groups (Group A, $359.25 \pm 17.91 \mathrm{~N}$; Group B, $374.38 \pm 13.75 \mathrm{~N}, \mathrm{P}>0.05$ ) as specific Fig. 4. There were three cases of sutures pulling through the tendon and five cases of the knotless-anchor loosen or pulled out regarding the single knotlessanchor modal of failure. However, in Group B, the Conversion repair (double-row) was presented, as were seven cases of sutures pulling through the tendon and one knotless-anchor loosen.

\section{Discussion}

Our results reveal no significant difference between group $A$ and $B$ concerning characters of the $10 \mathrm{~mm}$ and $15 \mathrm{~mm}$ supraspinatus tendon displacement and load to failure test. This displacement also indicates a single knotless-anchor for Bursal-side Ellman III PTRCTs is a useful technique that we could choose. This is the first study presenting a similar biomechanical effect with a double row to the best of our knowledge.

As far as we all know, DR rotator cuff repair is the most common and widely used clinical technique. Current literature works demonstrate that the biomechanical properties of a DR rotator cuff repair are superior to a single-row repair (SR). Additionally, it is evident from the reviewed researches that the DR technique has reconstructed the anatomic footprint of the rotator cuff significantly better than SR repairs $[18,19]$. Compared with the DR group's load to failure results, there was no significant difference between the two groups $(P>0.05)$. This also revealed the single Knotless-anchor with 2 Ethicon $2 \#$ could also reconstruct an anatomic footprint and provide biomechanical properties as DR rotator cuff repair. Moreover, regarding the $10 \mathrm{~mm}$ and $15 \mathrm{~mm}$ displacement of the supraspinatus, our results demonstrate a single Knotless-anchor could withstand the horizontal traction of biomechanical power to achieve a 
balance. On the other hand, concerning the supraspinatus displacement on $3 \mathrm{~mm}$ and $5 \mathrm{~mm}$, the DR group was presented superior biomechanical properties. This finding revealed that the suture anchor in the medial-row provided a firm power and reduced the medial rotator cuff's micro-motion.

Although the single knotless-anchor for Bursal-side Ellman III PTRCTs with two Ethicon 2\# is a simple, cheap, and effective technique, the essence of the substance of our method is an in situ repair (transtendon repair). To be specific, compared with the conversion repair with DR, Gonzalez-Lomas demonstrated that the ultimate failure load was significantly higher in the transtendon repair group [20]. Other primary research also indicated that transtendon repair was presented a higher ultimate failure load than double-row repair following full-thickness conversion [21]. These studies have similar effects on my findings, which also indicate the biomechanical advantages of tendon repair. Moreover, clinically transtendon repair has presented to be more effective than conversion to a full-thickness tear with the subsequent repair. Hytham Salem demonstrated that an in situ repair technique with double-row suture anchors in repairing bursal-sided PTRCTs provided a better fixation of the rotator cuff tissue while preserving the anatomy of the medial footprint [22]. This is a good study, which inspires me to start such research. Therefore, our technique for Bursal-side Ellman III PTRCTs is a simple, cheap, and effective technique that we could choose.

There are some surgical operation indications that we should follow by the use of this technique. There were three cases of sutures pulling through the tendon [23]. This revealed that 2 Ethicon 2\# sutures had a higher risk of cutting supraspinatus. Furthermore, theoretically, this might increase the failure of fixation or re-rupture rates. On the other hand, Ethicon 2\# sutures may cover weak areas in the tendon and decrease load to failure test. Therefore, the failure test does not simulate the natural failure biomechanics. So, several surgical indications of this method were mandatory: (i)Patients cannot bear the medical expenses and have cheap health insurance; (ii) Patients have a good elasticity or high quality of the supraspinatus under the arthroscopic debridement; (iii) The lateral row part of greater tuberosity bone density is normal;(iv) Patients have younger age than 60 years.

To sum up, there are various advantage of ours method as follows: (i) The bursal layer-only repair could produce the same clinical outcomes or structural integrity all-layers repair [24]; (ii) We retain the superior capsule, which provides the superior glenohumeral stability and has often been overlooked [25]; (iii) We also retain the cable(Fig. 2.C), which is the primary load-bearing structure within the supraspinatus [26]; (iv) The single knotless-anchor for Bursal-side Ellman III PTRCTs is a simple, cheap, and effective technique that benefit both doctors and patients; $(\mathrm{v})$ It is no necessary to consider the problem of the osteoporosis in the medial row.

However, our study also had some limitations. Firstly, with the lack of human samples, we only employed some 16 fresh-frozen specimens. We will employ more cadaveric shoulders in a future study. Secondly, the MRI should be employed to assess the specimen modal. Thirdly, with a limited number of available cadaver specimens, not all known fixation techniques such as a standard suture bridge were used in our study. Fourthly, the lesion of the cable group should employ for the next research. 


\section{Conclusion}

In conclusion, with the practical considerations of our hometown, the South-west of China, millions of patients cannot bear medical expenses and have cheap health insurance. So, the single knotless-anchor with two Ethicon 2\# for Bursal-side Ellman III PTRCTs is a simple, cheap, and effective technique that we could choose.

\section{Abbreviation}

PTRCTs: Bursal-side partial thickness rotator cuff tears; DR: Double-row; SR: Single-row repair

\section{Declarations}

\section{Acknowledgments}

Not applicable.

\section{Funding}

This study was supported by the Modern design and cultural research project of the research base on philosophy and Social Sciences of Sichuan in 2018 (Number: MD18E014) and Academician Workstation Construction Project of Lu zhou, Sichuan Province, China (No. 20180101).

\section{Availability of data and materials}

All data generated or analysed during this study are included in this published article and its supplementary information files.

\section{Author's contributions}

LG conducted the researches, wrote and revised the draft manuscript, and subsequent manuscript. ZBL and ZQ designed the study, revised the draft manuscript. GXG conducted the study, processed the figures, and participated in writing the draft manuscript. LY and QB were contributing to the statistical analysis, the manuscript submission. XP and LT were contributing to the literature search. FSJ contributed the funds collection, and study design. All authors read and approved the final manuscript.

\section{Ethics approval and consent to participate}

All the procedures were approved by the Ethical Inspection Committee at Southwest Medical University (KYT20180601).

\section{Consent for publication}

Not applicable. 


\section{Competing interests}

The authors declare that they have no competing interests.

\section{References}

1. Fukuda H, Hamada K, Yamanaka K. Pathology and pathogenesis of bursal-side rotator cuff tears viewed from en-bloc histologic sections. Clin Orthop Relat Res, 1990; (254): 75-80.

2. Matava MJ, Purcell DB, Rudzki JR. Partial-thickness rotator cuff tears. Am J Sports Med, 2005; 33(9): 1405-1417.

3. Fukuda H. Partial-thickness rotator cuff tears: a modern view on Codman's classic. J Shoulder Elbow Surg, 2000; 9(2): 163-168.

4. Herrmann SJ, Izadpanah K, Südkamp NP, et al. Tears of the rotator cuff. Causes--diagnosistreatment. Acta Chir Orthop Traumatol Cech, 2014; 81(4): 256-2666.

5. Milgrom C, Schaffler M, Gilbert S, et al. Rotator-cuff changes in asymptomatic adults. The effect of age, hand dominance and gender. J Bone Joint Surg Br, 1995; 77(2): 296-8.

6. Ellman H. Diagnosis and treatment of incomplete rotator cuff tears. Clin Orthop Relat Res, 1990; (254): 64-74.

7. Weber SC. Arthroscopic debridement and acromioplasty versus mini-open repair in the treatment of significant partial-thickness rotator cuff tears. Arthroscopy, 1999; 15(2): 126-131.

8. Eric JS, Michael JS, James K, et al. Multimedia article. The arthroscopic management of partialthickness rotator cuff tears: a systematic review of the literature. Arthroscopy, 2011; 27(4): 568-580.

9. Katthagen JC, Bucci G, Moatshe G, et al. Improved outcomes with arthroscopic repair of partialthickness rotator cuff tears: a systematic review. Knee Surg Sports Traumatol Arthrosc, 2018; 26(1): 113-124.

10. MacDonald P, McRae S, Leiter J, et al. Arthroscopic rotator cuff repair with and without acromioplasty in the treatment of full-thickness rotator cuff tears: a multicenter, randomized controlled trial. J Bone Joint Surg Am, 2011; 93(21): 1953-60.

11. lyengar JJ, Porat $S$, Burnett KR, et al. Magnetic resonance imaging tendon integrity assessment after arthroscopic partial-thickness rotator cuff repair. Arthroscopy, 2011; 27(3): 306-13.

12. Waibl B, Buess E. Partial-thickness articular surface supraspinatus tears: a new transtendon suture technique. Arthroscopy, 2005; 21(3): 376-81.

13. Castagna A, Rose GD, Conti1 M, et al. Predictive factors of subtle residual shoulder symptoms after transtendinous arthroscopic cuff repair: a clinical study. Am J Sports Med, 2009; 37(1): 103-8.

14. Kim KC, Shin HD, Cha SM, et al: Repair integrity and functional outcome after arthroscopic conversion to a full-thickness rotator cuff tear: articular-versus bursal-side partial tears. Am J Sports Med, 2014; 42(2): 451-456. 
15. Cordasco FA, Backer M, Craig EV, et al. The partial-thickness rotator cuff tear: is acromioplasty without repair sufficient? Am J Sports Med, 2002; 30(2): 257-60.

16. Yang S, Park HS, Flores $S$, et al. Biomechanical analysis of bursal-sided partial thickness rotator cuff tears. J Shoulder Elbow Surg, 2009; 18 (3): 379-85.

17. Barber FA, Drew OR. A biomechanical comparison of tendon-bone interface motion and cyclic loading between single-row, triple-loaded cuff repairs and double-row, suture-tape cuff repairs using bio-composite anchors. Arthroscopy, 2012; 28(9): 1197-1205.

18. Wall LB, Keener JD, Brophy RH. Double-row vs single-row rotator cuff repair: a review of the biomechanical evidence. J Shoulder Elbow Surg, 2009; 18 (6): 933-41.

19. Smith CD, Alexander S, Hill AM, et al. A biomechanical comparison of single and double-row fixation in arthroscopic rotator cuff repair. J Bone Joint Surg Am, 2006; 88(11): 2425-31.

20. Gonzalez-Lomas G, Kippe MA, Brown GD, et al. In situ transtendon repair outperforms tear completion and repair for partial articular-sided supraspinatus tendon tears. J Shoulder Elbow Surg, 2008; 17 (5): 722-8.

21. Park MC, Jun BJ, Park CJ, et al. Biomechanical analysis of a knotless transtendon interimplant mattress repair for partial-thickness articular-sided rotator cuff tears. Am J Sports Med, 2009; 37(12): 2427-34.

22. Salem H, Carter A, Tjoumakaris F, et al. Double-Row Repair Technique for Bursal-Sided PartialThickness Rotator Cuff Tears. Arthrosc Tech, 2018; 7(3): 199-203.

23. Peters KS, Lam PH, Murrell GAC. Repair of partial-thickness rotator cuff tears: a biomechanical analysis of footprint contact pressure and strength in an ovine model. Arthroscopy, 2010; 26(7): 87784.

24. Kim SJ, Choi YR, Lee HH, et al. Surgical Results of Delaminated Rotator Cuff Repair Using SutureBridge Technique with All-Layers or Bursal Layer-Only Repair. Am J Sports Med, 2016; 44(2): 468-73.

25. Adams CR, DeMartino AM, Rego G, et al. The Rotator Cuff and the Superior Capsule: Why We Need Both. Arthroscopy, 2016; 32(12): 2628-2637.

26. Mesiha MM, Derwin KA, Sibole SC, et al. The biomechanical relevance of anterior rotator cuff cable tears in a cadaveric shoulder model. J Bone Joint Surg Am, 2013; 95(20): 1817-24.

\section{Figures}



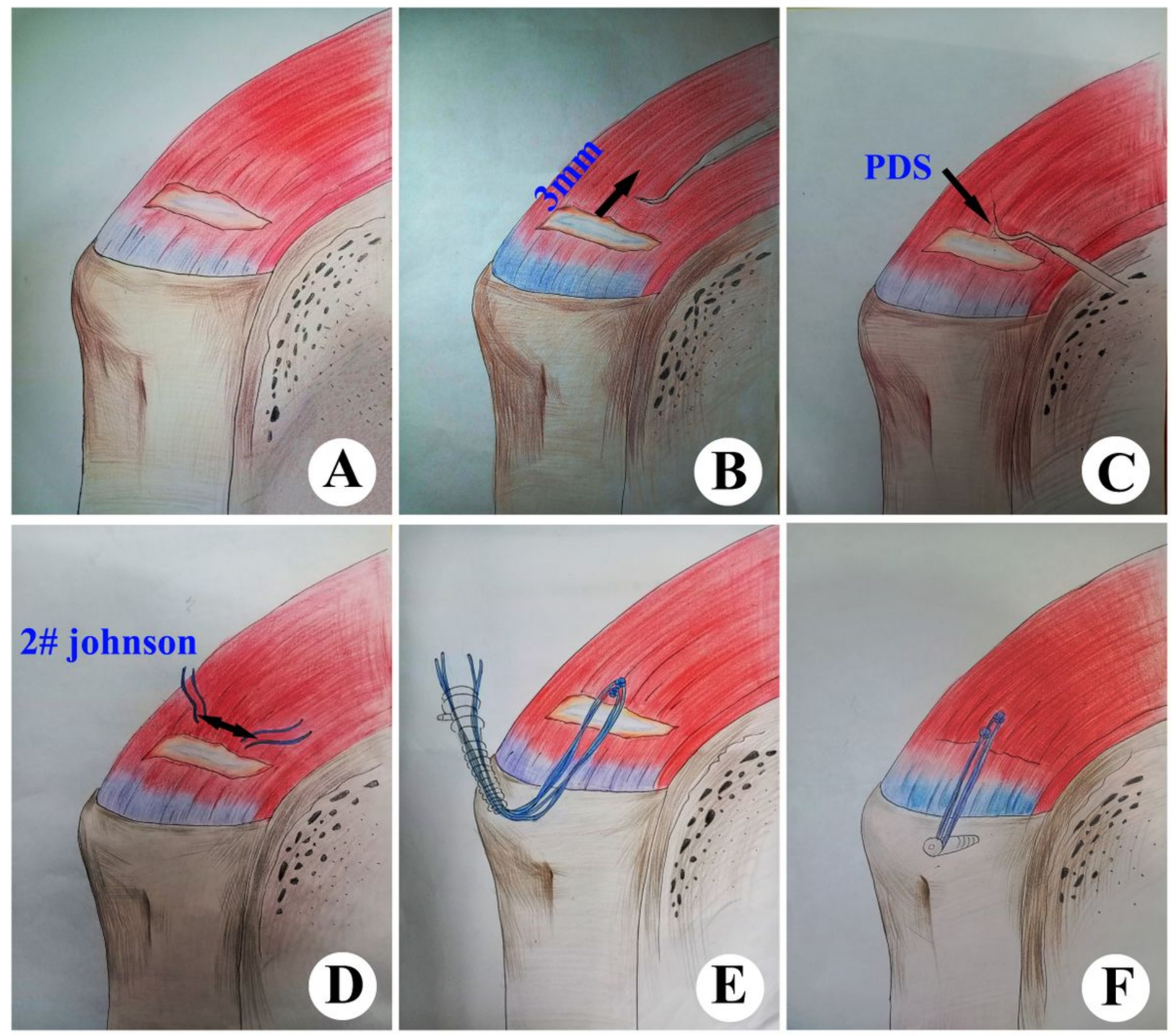

\section{Figure 1}

The Single Knotless-anchor technique of Sketch models. (A) 75\% Bursal-side PTRCTs. (B) The distance of a suture hook to puncture. (C) The PDS thread was passing through the hook. (D) The Ethicon $2 \#$ thread was passing through the hook. (E) The 2 Ethicon $2 \#$ thread was passing through the Knotless-anchor. (F) The fixation of Single Knotless-anchor. 

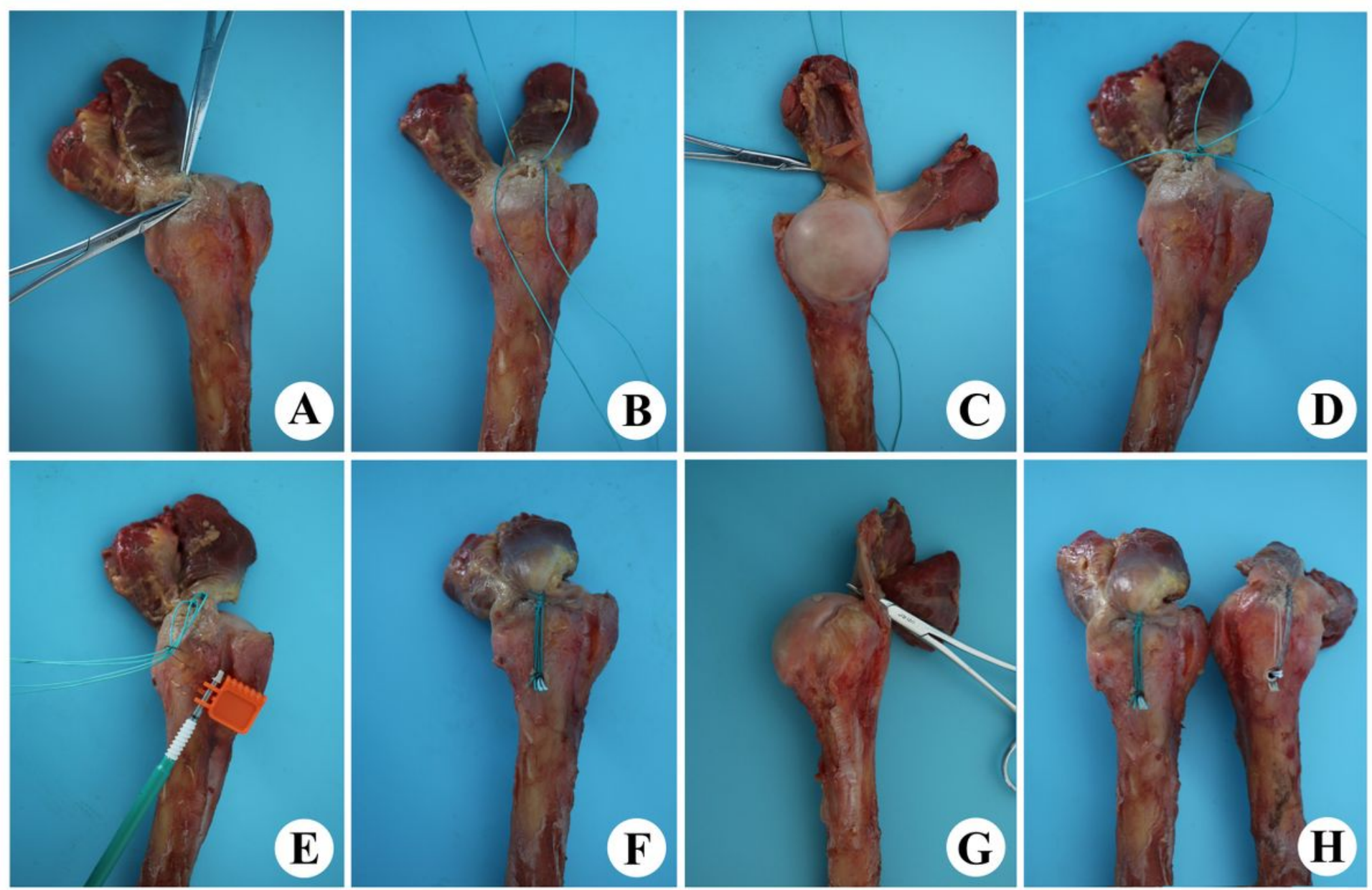

Figure 2

The specific process of two model repair techniques. (A) 75\% Bursal-side PTRCTs with blade and bone contusion. (B) The 2 Ethicon 2\# thread suture through the supraspinatus. (C) Retain the superior capsule and cable. (D) Knot the2 Ethicon 2\# thread. (E) Passing through the Ethicon 2\# to Knotless-anchor and making sure the location of the lateral row. $(F)$ The fixation of Single Knotless-anchor. (G)The conversion modal involved completing a PTRCTs to a full-thickness rotator cuff tear. $(H)$ The fixation of the conversion repair with double-row. 

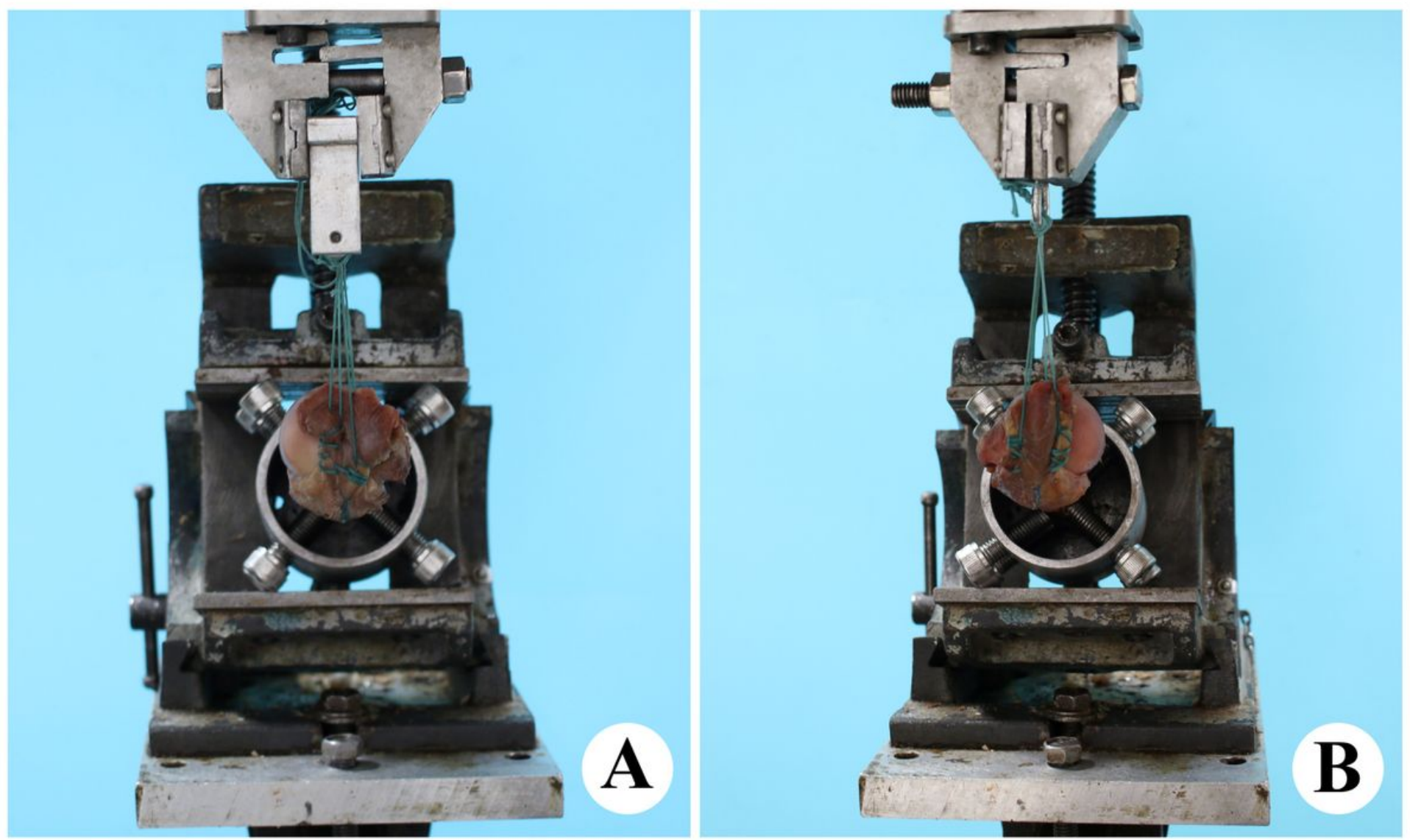

\section{Figure 3}

The load test and load-to-failure test. (A) Group A, the single knotless-anchor repair with two Ethicon 2\#. (B) Group B, the conversion repair with double-row. 


\section{Load to failure}

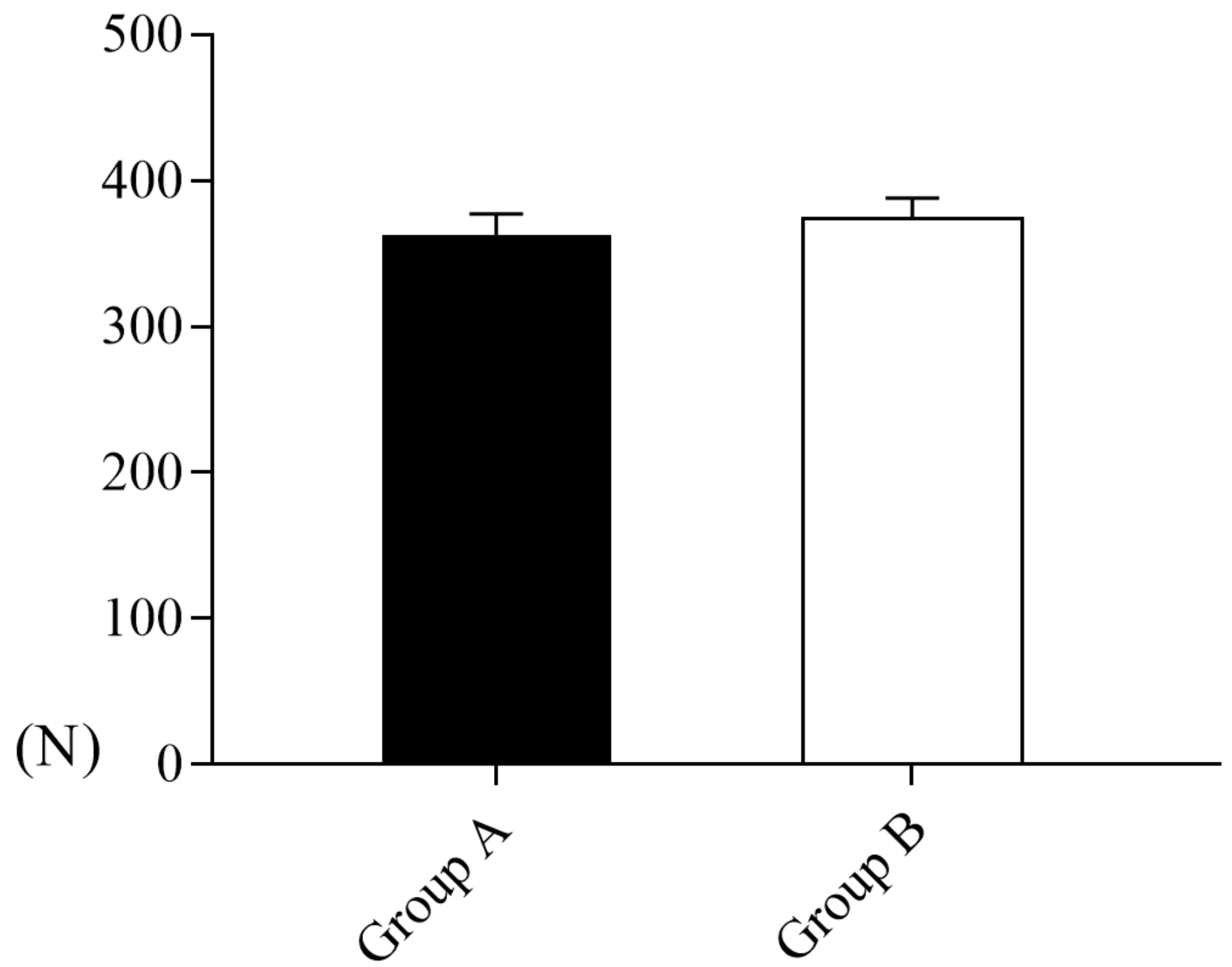

Figure 4

The result of the load-to-failure test. There was no significant difference between the two groups (P凶0.05).

\section{Supplementary Files}

This is a list of supplementary files associated with this preprint. Click to download. 
- BursalsideRCTEthicallnspection.pdf

- FailureSingleKnotlessanchor.csv

- Statisticalprocess.xIsx

- renamed09e28.csv 